\title{
Gobernanza del agua y Consejos de cuenca: análisis desde los derechos humanos al agua y a la participación ambiental ${ }^{*}$
}

\author{
Recibido: 09 de abril de 2019 • Aprobado: 22 de mayo de 2019 \\ https://doi.org/10.22395/ojum.v18n37al \\ Érika Castro-Buitrago*" \\ Juliana Vélez-Echeverri" \\ Mauricio Madrigal-Pérez ${ }^{* * *}$
}

\begin{abstract}
RESUMEN
La introducción de la gobernanza del agua en el ordenamiento colombiano y la alta conflictividad socioecológica motivan los análisis que vinculan la naturaleza con los derechos humanos. Desde una investigación de corte dogmático descriptivo y analítico, este artículo propone el relacionamiento de la gobernanza del agua con los derechos humanos por medio de la integración de una serie de estándares jurídicos vinculados al Acuerdo de Escazú y al derecho humano al agua. Entre los hallazgos se identificaron: la deficiente reglamentación de la participación y del acceso a la información, la falta del financiamiento público, la limitación de la deliberación, la desmotivación de la concertación y la falta de integración efectiva de los grupos en condición de vulnerabilidad. Los objetivos de la gobernanza del agua, materializados en los Consejos de cuenca, se relacionan con los estándares jurídicos integrados y los hallazgos obtenidos.

Palabras clave: gobernanza del agua; derecho humano al agua; principio 10; Tratado de Escazú; Consejos de cuenca.
\end{abstract}

\footnotetext{
Este artículo presenta resultados del proyecto investigación titulado Gobernanza del agua y derechos humanos financiado por el Centro Latinoamericano de Estudios Ambientales -Celeam-, al que pertenece el Grupo de Investigación en Derecho Ambiental Latinoamericano y corresponde a la línea de investigación Derecho ambiental, gobernanza y derechos humanos. Inicio: julio de 2017, culminación: diciembre de 2018. En colaboración con la línea de investigación en derecho ambiental de la Universidad de Medellín y la línea de investigación en derecho ambiental de la Institución Universitaria de Envigado, para ser sometido a proceso de evaluación interna y externa.

** Abogada y especialista en Derecho Ambiental, Colegio Mayor Nuestra Señora del Rosario, Bogotá, Colombia; doctora en Ordenación del Territorio y Medio Ambiente, Universidad Autónoma de Madrid, Madrid, España. Directora de la línea de investigación en derecho ambiental, Facultad de Derecho de la Universidad de Medellín, Medellín, Colombia. Correo electrónico: ecastro@udem.edu.co

${ }^{* * *}$ Abogada, Universidad de Medellín, Medellín, Colombia; especialista en Derecho Ambiental, Universidad Externado de Colombia, Bogotá, Colombia. Investigadora del Centro Latinoamericano de Estudios Ambientales-Celeam-, Armenia, Colombia. Correo electrónico: jvelez@celeam.org

*.** Abogado, Universidad de Medellín, Medellín, Colombia; especialista en Derecho Ambiental, Universidad Externado de Colombia, Bogotá, Colombia; magíster en Derecho, Universidad de Antioquia. Coordinador de la línea de investigación en Derecho Ambiental, Institución Universitaria de Envigado, Envigado, Colombia; director del Centro Latinoamericano de Estudios Ambientales-Celeam-, Armenia, Colombia. Correo electrónico: mfmadrigal@correo.iue.edu.co
} 


\title{
Water Governance and Basin Councils: an Analysis from Human Rights to Water and Environmental Participation
}

\begin{abstract}
The introduction of water governance to the Colombian ordering and the high sociological potential for conflict are the foundation of those analyses which links nature with human rights. From a dogmatic-descriptive and analytical research perspective, this articles proposes a link between water governance with human rights via the integration of a series of law standards linked to the Escazú Agreement and the human right to water, Among the findings, the research identified: a deficient ruling of participation and information access, a lack of knowledge from the public, some limitation on deliberation and demotivation for agreements, as well as a lack of effective integration of groups in vulnerable condition. The objectives of governance of water materialized in the basin councils, are related to integrated law standards and the research findings.
\end{abstract}

Keywords: water governance; human right to water; 10th principle; Escazú Agreement; Basin councils.

\section{Governança da água e Conselhos de Bacia: análise a partir dos Direitos Humanos à água e à participação ambiental}

\section{RESUMO}

A introdução da governança da água no ordenamento colombiano e a alta conflitividade socioecológica motivam as análises que vinculam a natureza com os Direitos Humanos. A partir de uma pesquisa de corte dogmático descritivo e analítico, este artigo propõe a relação da governança da água com os Direitos Humanos por meio da integração de parâmetros jurídicos vinculados ao Acordo de Escazú e ao Direito Humano à água. Entre os achados, foram identificadas: a deficiente regulamentação da participação e do acesso à informação, a falta do financiamento público, a limitação da deliberação, a desmotivação do acordo e a falta de integração efetiva dos grupos em condição de vulnerabilidade. Os objetivos da governança da água, materializados nos Conselhos de Bacia, estão relacionados com os parâmetros jurídicos integrados e os achados obtidos.

Palavras-chave: governança da água; Direito Humano à água; princípio 10; Tratado de Escazú; Conselhos de Bacia. 


\section{INTRODUCCIÓN}

A inicios del milenio, la Cepal alertaba sobre los obstáculos que Latinoamérica y el Caribe enfrentaban para alcanzar una gestión integrada del agua. Entre todos ellos, los principales son: "la carencia de sistemas de articulación" (Dourojeanni y Jouravlev, 2001, p. 7) interdisciplinaria de conocimientos entre ciencias duras y blandas, la participación de las poblaciones y la falta de transparencia pública (Dourojeanni y Jouravlev, 2001, pp. 7-8). Hasta el año 2010, con la política hídrica nacional (PHN) (Ministerio de Ambiente, Vivienda y Desarrollo Territorial, 2010) y más concretamente en 2012 con la creación de los Consejos de cuenca, el Gobierno colombiano formuló una estrategia que le apuesta a hacerle frente al obstáculo planteado por la Cepal y que encuentra fundamento en el concepto de gobernanza del agua.

Como antecedente de la creación jurídica de los Consejos de cuenca, se encuentra el Decreto 2857 de 1981, que fue el primer instrumento que reguló un esquema de planificación hídrica en Colombia. Esta norma derogada por el Decreto 1729 de 2002 se ajustó al Sistema Nacional Ambiental y fue creado por la Ley 99 de 1993. Posteriormente, fue derogado por el Decreto 1640 de 2012, vigente actualmente. Este último tiene como objetivo central desarrollar el nuevo esquema de planificación hídrica para las cuencas, microcuencas y acuíferos establecido en el 2010 por la PHN (Ministerio de Ambiente y Desarrollo Sostenible, 2012).

Este esquema de planificación hídrica tuvo como novedad la inclusión de la gobernanza del agua y la gestión del riesgo como componentes transversales, cuya motivación fue el preocupante contexto socioambiental que vivió el país en las olas invernales consecutivas de los años 2008, 2009, 2010 y 2011 consideradas las más graves de la historia nacional. En efecto, a finales del 2010, como consecuencia del fenómeno de La Niña, el país enfrentó una verdadera tragedia:

246 muertos, 246 heridos y 99 desaparecidos. Las lluvias afectaron a más de 400 mil familias en más de 650 municipios de 28 de los 32 departamentos del país, donde el invierno destruyó 2.938 viviendas y dejó otras 296.340 en malas condiciones (Revista Semana, 2010).

A lo anterior debe añadirse la alta contaminación del agua, especialmente en nueve ciudades principales: Bogotá, Cartagena, Barranquilla, Bucaramanga, Cali, Medellín, Pereira y Cúcuta, lo que ha fundamentado que los estudios nacionales sobre los recursos hídricos señalen que aproximadamente el 50 \% del agua en Colombia es de mala calidad y que en promedio la carga vertida de mercurio es de 205 toneladas al año (Ideam, 2015).

Como parte de las soluciones propuestas en el plan de acción de la PHN se crean los Consejos de cuenca, como el principal espacio de participación en la ordenación 
y manejo de cuencas'. En cumplimiento del citado plan, para noviembre de 2018 se habían conformado 95 Consejos de cuenca, de conformidad con lo establecido en la Resolución 509 de 2013 (Ministerio de Ambiente y Desarrollo Sostenible, 2018). Es decir, el 70,3 \% de las 135 cuencas priorizadas (entre las 309 existentes el país) para desarrollar un Plan de Ordenamiento y Manejo de Cuenca (en adelante, Pomca) (IGAC, 2014). De esta manera, y como mandato de la PHN, los Consejos de cuenca se convirtieron en la principal expresión jurídica y práctica de la gobernanza del agua en el país, orientados, como se mencionó, a la participación de los actores sociales en el desarrollo de los Pomca, instrumentos que tienen mayor jerarquía que los planes de ordenamiento territorial.

Sin embargo, el concepto de gobernanza del agua ha sido criticado por académicos y organizaciones de la sociedad civil. Uno de los aspectos más discutidos está relacionado con la no consideración de criterios de multiculturalidad e interculturalidad y la falta de atención prioritaria a los grupos en condición de vulnerabilidad en el marco de los derechos humanos, como lo señalan De Sousa Santos y Rodríguez (2007) quienes afirman que, para los partidarios de la gobernanza pragmatista, las asimetrías del poder entre actores sociales no son tan profundas como para impedir este tipo de procesos. Con acento en estas afirmaciones, Querejazu (2016) indica que el concepto de la gobernanza y el proyecto político que lo cobija:

Perpetúa la exclusión a través de violencias encubiertas que habilita ciertas formas de ser y estar en el mundo, entre ellas, procesos de construcción del otro, la aceptación de una manera de conocer la realidad, restringiendo la posibilidad de pensar lo político global por otras vías (p. 148).

Pese a los fuertes cuestionamientos, existen propuestas de investigación que, manteniendo una postura crítica, valoran la posibilidad de la gobernanza como un medio para garantizar los derechos humanos y para ello proponen, a partir de las críticas, el desarrollo de enfoques alternativos para el análisis de la gobernanza. Ejemplo de ello es la investigación de Madrigal (2018) denominada Enfoque alternativo de la gobernanza hídrica que relaciona la gobernanza con los derechos humanos. Esta propuesta se fortalece en un nuevo escenario político-jurídico en el que, con la aprobación del Acuerdo de Escazú (Naciones Unidas, 2018) se establecen los estándares mínimos a los que debería ajustarse la gobernanza ambiental, por lo menos en Latinoamérica y el Caribe.

Considerando lo anterior, la presente investigación tiene como objetivo general contribuir con el mejoramiento del contenido normativo de la gobernanza del agua

Los Consejos de cuenca hacen parte del eje transversal de la gobernanza del agua (es una interpretación del contenido de la política hídrica que en el objetivo de "gobernabilidad" desarrolla los Consejos de cuenca). 
desde el enfoque de los derechos humanos. Para cumplir con los propósitos antes mencionados, se aborda primero el estudio del concepto de gobernanza del agua con énfasis en las propuestas desarrolladas en Iberoamérica, brindando una aproximación al estado del arte que tiene como criterio diferenciador la integración de los derechos humanos en el análisis de la gobernanza del agua, y que finaliza con la elección del referente teórico. En un segundo momento, de acuerdo con el referente teórico elegido, se analiza el marco jurídico de la gobernanza del agua en Colombia, con énfasis en lo contenido en el Decreto 1640 de 2012, en la Resolución 509 de 2013 y en la guía para la elaboración de los Pomca del año 2014. Por último, como conclusión se relacionan los objetivos de la gobernanza del agua y de los Consejos de cuenca -consagrados en el marco jurídico con el enfoque propuesto-y los hallazgos obtenidos.

\section{1. МЕ́TODO}

La investigación base de este trabajo es de corte dogmático descriptivo y analítico. Dogmático en tanto tiene como objeto de investigación todas las expresiones del derecho, tomando en cuenta su encaje con la realidad, la existencia o la necesidad de los presupuestos y condiciones para su vinculación a sus máximas, las cuales son los fines, los principios y los derechos.

La investigación está integrada por tres momentos: el primero está vinculado con la dimensión descriptiva. Se caracterizan los principales desarrollos teóricos sobre la gobernanza del agua por medio de la revisión documental detallada y con base en los siguientes criterios: i) tipo de información: artículos, informes y libros de investigación; ii) período de consulta: 2000-2018; iii) bases de datos: Dialnet, Scielo y Google Académico; iv) ámbito: iberoamericano; v) área del conocimiento: derecho y vi) información recopilada: conceptualización, metodología y conclusiones. Una vez caracterizado el concepto de gobernanza se describe el enfoque teórico elegido.

En el segundo momento se formula el enfoque por medio del cual se analizará el marco jurídico de la gobernanza del agua, integrado por la propuesta de Madrigal (2018) y los estándares del Acuerdo de Escazú y del derecho humano al agua. En ese sentido, se proyecta la gobernanza desde el enfoque de derechos humanos.

En el tercer y último momento se realizó el análisis del contexto jurídico sobre la gobernanza del agua en Colombia, se valoraron los vacíos e inconsistencias de las normas jurídicas desde el enfoque propuesto, en particular los aspectos vinculados con la deliberación, la transparencia y rendición de cuentas, el acceso a la información y la autonomía. 


\section{CARACTERIZACIÓN DE LA GOBERNANZA DEL AGUA}

El concepto de gobernanza ha sido calificado como histórico y dinámico por diversos autores. En términos generales, la mayoría lo ve como un nuevo modelo de gobernar, más cooperativo entre los actores públicos y los actores privados/sociales, concepto que difiere del de gobernabilidad centrado en el gobernante y su capacidad directiva (Torres y Ramos, 2012).

En la evolución histórica del concepto se identifican dos grandes enfoques: el primero está orientado a la descripción de los cambios en las formas de gobernación y el segundo asume la gobernanza en términos prescriptivos, en el campo de las organizaciones internacionales como buena gobernanza, según el cual se entiende como un modelo ideal de gobernar que contiene una serie de principios o directrices a ser observados (Castro-Buitrago, 2017, p. 147). Aunque existe una vasta literatura sobre el tema, cabe citar como principal hito el Libro blanco sobre la gobernanza europea preparado por la Comisión Europea (2001), en el que se hace una lista de principios para mejorar la apertura en la formulación de las políticas públicas.

En Latinoamérica se destaca el estudio del mexicano Francisco Porras (2016, p. 113), en el cual menciona que la gobernanza tiene una formidable polisemia, originada, entre otras causas, en "una cierta desvinculación entre el desarrollo teórico de las nociones y las definiciones propuestas y el sustrato ontológico que las sostiene". La calidad polisémica y de indeterminación teórica de la gobernanza se refleja en la identificación y descripción de 61 definiciones desarrolladas por Porras, las cuales son descritas en lo que denomina "mapa de literatura sobre gobernanza", integrado por dos grandes orientaciones. Las primeras abordan la gobernanza con un mayor grado de abstracción en temas como las relaciones internacionales y las esferas territoriales. Las segundas se relacionan con las redes y por lo tanto hacen énfasis en aspectos como los actores y en lo normativo-democrático.

Ante la multiplicidad de enfoques y contenidos en el ámbito académico, algunos autores como Cerrillo (2005) han sugerido claves para evaluar el contenido y calidad de la gobernanza con la formulación de algunos objetivos a los que tributan los principios de la gobernanza²: i) que estén todos los actores que han de estar, cada uno de ellos asumiendo el rol que le corresponde; ii) que los actores tengan las condiciones necesarias y suficientes para poder tomar las decisiones que les correspondan, y iii) que se puedan tomar decisiones (Cerrillo, 2005, p. 19). No obstante, cabe advertir que la misma historia del concepto enseña que establecer un contenido e indicadores de calidad de la gobernanza debe corresponder a un ejercicio explicativo del contexto

Estos principios son: participación, transparencia, rendición de cuentas, coherencia (entre las políticas públicas y normas) y eficacia (Cerrillo, 2005, p. 22). 
jurídico-político y social interno. Este desconocimiento puede ocasionar un mero trasplante de definiciones, lo que ha sido una de las principales críticas de la gobernanza porque no constituye un aporte a la solución de los problemas que, justamente, debería encarar que, como en el caso de la gobernanza del agua, se dirige a una toma de decisiones más informada y participativa de todos los actores para alcanzar los fines más altos de protección de derechos.

En ese orden de ideas, un gran número de autores afirma que la complejidad de la noción de gobernanza del agua depende del contexto y orientación política del autor o institución. La revisión documental arrojó los siguientes resultados generales:

- En torno al rastreo en las bases de datos bajo la categoría gobernanza del agua, Google académico (1.220), Dialnet (380) y Scielo (44).

- La existencia de subclasificaciones de la gobernanza del agua: gobernanza del agua residual (Pacheco-Vega, 2015), gobernanza deliberativa en la gestión de cuencas hidrográficas (Parés, Ballester, Espluga y Bruqué, 2014) y gobernanza de aguas subterráneas, (CuadradoQuesada, 2017).

- La identificación de 23 definiciones que, aunque contemplan diferentes enfoques y elementos, coinciden en la integración de actores no gubernamentales en la toma de decisiones en asuntos hídricos de carácter público.

Las definiciones de la gobernanza del agua identificadas pueden integrarse en dos enfoques principales: el primero, descriptivo de la gobernanza como proceso y nueva forma de gobierno, con énfasis en las relaciones entre actores y los flujos de poder, y el segundo, prescriptivo, como un modelo de buen gobierno identificable con las categorías de buena gobernanza o gobernanza democrática, relacionado por lo tanto con aspectos normativos.

Del primer enfoque hacen parte las definiciones como las desarrollados por la FAO (2014), Ballester y La Calle (2015), Domínguez (2006), Mussetta (2009) y Santa (2014). Entre todas, destacan la definición de Mello y Villar (2015, p. 55) para quienes la gobernanza del agua "es un proceso político en el cual diversos actores debaten e intentan llegar a un acuerdo acerca de los valores, principios, objetivos a lograr e instrumentos que se usarán para la gestión", y lo propuesto por Madrigal (2018), quien describe la gobernanza del agua como un "proceso social y dinámico de integración de saberes en la toma de decisiones políticas, normativas y jurídicas, por medio del cual se pretende gestionar equitativa y territorialmente el patrimonio hídrico con el fin de garantizar el derecho humano al agua" (p. 43). Esta última definición integra un elemento esencial: el relacionamiento entre la gobernanza con los derechos humanos. 
Por su parte, el segundo enfoque prescriptivo ha sido promovido por las organizaciones internacionales o intergubernamentales, se destaca la Organización para la Cooperación y Desarrollo Económicos (OCDE), el Banco Mundial y el Consejo Mundial del Agua. Este último, con referencia a la gestión integral del recurso (GIRH) afirma que:

Una buena gobernanza en la GIRH es aquella donde la autoridad juega un papel menos protagónico, deja actuar en aquellos espacios donde son más eficientes otros actores, o ejerce un liderazgo que permita establecer reglas claras que faciliten la participación de otros actores y sectores en la gestión del agua, donde las decisiones respecto a las políticas públicas son establecidas por consenso con todos los actores que intervienen en dicha gestión del agua (Consejo Mundial del Agua 2012, p. 11).

Otra definición de este enfoque, proveniente de la academia, considera que "una buena gobernanza del agua presupone una política participativa y un proceso decisorio abierto en lo que al uso del agua se refiere, descentralizado, con transferencia de poder para el gobierno local” (Jacobi, Silva-Sánchez y Fracalanza, 2015, p. 46).

Ambas perspectivas tienen como eje la participación en su doble faceta (derechodeber). En las definiciones propuestas por el segundo enfoque, se hace más énfasis en la participación como un deber e integra conceptos como la corresponsabilidad; en cambio, en el primer enfoque se identifica una definición que vincula el proceso de gobernanza del agua con los derechos humanos, con lo que se busca superar los puntos críticos de la gobernanza, como la deficiente valoración tanto de los sujetos y grupos en condición de vulnerabilidad como de la participación como un derecho.

\section{LA GOBERNANZA DEL AGUA DESDE EL ENFOQUE DE LOS DERECHOS HUMANOS}

El punto de partida es el enfoque alternativo de la gobernanza del agua propuesto por Madrigal (2018) para el desarrollo de un estudio de caso, específicamente el proceso de gobernanza hídrica en la cuenca del río Aburrá con énfasis en el Consejo de cuenca. El enfoque sirve de base para analizar el marco jurídico de la gobernanza del agua en Colombia, pues la misma integra de manera dialéctica dos aspectos claves: el derecho humano al agua y los grupos en condición de vulnerabilidad. El primero lo describe de manera amplia, complementa la visión tradicional vinculada al ámbito de los servicios públicos, puntualmente al agua potable y al saneamiento bajo criterios de disponibilidad, calidad, aceptabilidad y accesibilidad que buscan una garantía más particular e individual. A partir del reconocimiento de los anteriores criterios, desarrolla el carácter colectivo del derecho humano analizado, relacionado con la conservación y protección de las fuentes hídricas y la democratización y enlazando el mencionado derecho con la participación y, así con la gobernanza. 
En torno a la integración de los grupos en condición de vulnerabilidad, el enfoque incorpora algunos de los postulados de la ecología política del agua y valora la participación como un derecho humano amplio que comprende aspectos étnicos, de género, de acceso a la información, transparencia y rendición de cuentas y acciones afirmativas ${ }^{3}$.

Sobre el Acuerdo de Escazú, es preciso comenzar mencionando que todo enfoque que sea asumido para tratar la gobernanza del agua tendrá que responder a unos estándares jurídicos mínimos. En este caso, cabe señalar que el 4 de marzo de 2018 se aprobó, en la ciudad de Escazú, Costa Rica, el acuerdo regional sobre el acceso a la información, la participación pública y la justicia en asuntos ambientales en América Latina y el Caribe, llamado Acuerdo de Escazú. Este tratado negoció explícitamente las reglas de la gobernanza ambiental de la región y estableció los indicadores mínimos que un proceso democrático, pluralista y deliberativo de toma decisiones ambientales debe respetar.

Debe recordarse que el Acuerdo de Escazú es el resultado de los compromisos derivados de la Declaración sobre el Principio 10: acceso a la información, participación y justicia en asuntos ambientales en América Latina y el Caribe, adoptada en la Conferencia de las Naciones Unidas sobre el Desarrollo Sostenible de 2012 (Naciones Unidas, 2012). El proceso para la adopción del Acuerdo de Escazú tuvo una fase preparatoria entre los años 2012-2014 que culminó con el establecimiento del Comité de Negociación, lo cual a su vez marcó el inicio de la etapa de negociación en la cual se desarrollaron nueve reuniones en el periodo 2014-2018 (Cepal, 2018).

El mencionado proceso estuvo marcado, como lo describen Castro-Buitrago y Calderón (2018), por las propuestas de algunos Estados parte en las que se evidenció una clara intención de disminuir el grado de obligatoriedad del acuerdo, inclusive por debajo de los estándares mínimos reconocidos internacionalmente. Aunque en el texto preliminar del proyecto de acuerdo se incluían en detalle diferentes reglas procedimentales clave para los procesos deliberativos o de gobernanza, el texto final logró consignar los estándares mínimos de los derechos de acceso que al integrarse a los procesos de gobernanza del agua le brindan el contenido normativo suficiente para valorar la garantía del derecho humano al agua. En lo que atiende expresamente a los estándares para la garantía del derecho a la participación, en la siguiente tabla se incluye una síntesis de las reglas aprobadas en el artículo 7 del Acuerdo de Escazú (Naciones Unidas, 2018).

\footnotetext{
3 "Alude a todas aquellas medidas, políticas o decisiones públicas a través de las cuales se establece un trato ventajoso, y en cuanto tal formalmente desigual, que favorece a determinadas personas o grupos humanos tradicionalmente marginados o discriminados, con el único propósito de avanzar hacia la igualdad sustancial de todo el conglomerado social" (Corte Constitucional, 2010).
} 
Tabla 1. Participación pública en los procesos de toma de decisiones ambientales

1. Participación abierta e inclusiva en la toma de decisiones ambientales.

2. Mecanismos participativos que permitan: revisiones, reexaminaciones o actualizaciones en proyectos y actividades ambientales.

3. Promoción de la participación en los procesos de autorizaciones ambientales, elaboración de políticas, normas y reglamentos.
6. Medios y sistemas apropiados para informar de forma efectiva, comprensible y oportuna al público.

7. Posibilidad que el público presente observaciones por medios apropiados y disponibles antes de que la autoridad tome la decisión.

8. Adoptada la decisión debe informarse opor tunamente al público, incluyendo motivos y fundamentos que la sustentan y el modo en que se tuvieron en cuenta las observaciones del público.

4. Participación efectiva desde las etapas iniciales del proceso de toma de decisiones.

9. Promoción de la valoración del conocimiento local, el diálogo y la interacción de las diferentes visiones y saberes.

5. Plazos razonables en el procedimiento de participación para que exista tiempo suficiente de informar al público.

10. Identificación y apoyo a personas o grupos en condición de vulnerabilidad para involucrarlos de manera activa, oportuna y efectiva en los mecanismos de participación.

Fuente: elaboración propia a partir del contenido del Acuerdo de Escazú (Naciones Unidas, 2018).

Sobre los estándares del derecho humano al agua, el punto de partida es su fundamentación como derecho autónomo y esencial para la garantía de los demás derechos, que se encuentra en la Observación General n. 15 de 2002 del Comité de Derechos Económicos, Sociales y Culturales de las Naciones Unidas. La base de esta fundamentación es la interpretación de los artículos 11 y 12 del Pacto Internacional de Derechos Económicos, Sociales y Culturales en el sentido de considerar el derecho al agua como parte indiscutible de las condiciones esenciales para la supervivencia, "asociadas al más alto nivel posible de salud (párr. 1, artículo 12) y al derecho a una vivienda y una alimentación adecuadas (párr. 1, artículo 11)" (Comité de Derechos Económicos, Sociales y Culturales de las Naciones Unidas , 2002, p. 2).

Los servicios públicos de acueducto y alcantarillado han sido el eje central para la garantía del derecho al agua, tomando como referencia los criterios normativos básicos descritos en la Observación General n. 15:

a. Disponibilidad: El abastecimiento de agua de cada persona debe ser continuo y suficiente para los usos personales y domésticos (...); 6. Calidad: El agua necesaria para cada uso personal o doméstico debe ser salubre y, por lo tanto, no ha de contener microorganismos 
o sustancias químicas o radiactivas que puedan constituir una amenaza para la salud de las personas (...), y La accesibilidad. El agua y las instalaciones y servicios de agua deben ser accesibles a todos, sin discriminación alguna, dentro de la jurisdicción del Estado parte. La accesibilidad presenta cuatro dimensiones superpuestas: accesibilidad física (...), accesibilidad económica (...), no discriminación (...) y acceso a la información (...) (Comité de Derechos Económicos, Sociales y Culturales de las Naciones Unidas , 2002, pp. 5-7).

Existe un relacionamiento claro y evidente entre los criterios normativos y los servicios públicos descritos, pero no ocurre lo mismo con la gestión de cuencas hidrográficas y con los procesos de gobernanza del agua que allí se desarrollan. En ese sentido, la Federación Iberoamericana de Ombusman (FIO), en el XII informe sobre derechos humanos titulado Derecho al agua, señala que la eficacia de este derecho involucra cuestiones que van más allá del contenido normativo básico, como por ejemplo, la necesidad de la implantar un modelo de gestión integral del agua, que no solo garantice el acceso universal a este recurso, sino que promueva el control de la demanda, la unidad de cuenca, el buen estado ecológico de los ecosistemas y la gestión coordinada y pacífica de las fuentes transfronterizas (Federación Iberoamericana de Ombudsman, 2015, p. 40).

Considerando lo antes mencionado, existe una serie de estándares sobre el derecho humano al agua que enriquecen los procesos de gobernanza y se encuentran contenidos en la Observación General n. ${ }^{\circ} 15$ y en el Informe de la FIO y se presentan en la tabla 2.

Tabla 2. Estándares sobre el derecho humano al agua

1. Reconocimiento explícito del derecho humano al agua en el ordenamiento jurídico y político nacional.
6. Se suministre agua salubre suficiente a los grupos que tienen dificultades físicas para acceder al agua, como las personas de edad, los discapacitados, las víctimas de desastres naturales, las personas que viven en zonas propensas a desastres y las que viven en zonas áridas y semiáridas o en pequeñas islas.
2. No se excluya a las mujeres de los procesos de adopción de decisiones sobre los recursos y los derechos en materia de agua.
3. El acceso de los pueblos indígenas a los recursos de agua en sus tierras ancestrales sea protegido de toda transgresión y contaminación ilícitas.
7. Las zonas rurales y las zonas urbanas desfavorecidas tengan acceso a servicios de suministro de agua en buen estado de conservación.
8. Formular leyes y políticas nacionales que favorezcan el desarrollo de un sistema de gestión integral del agua, que incluya todas las dimensiones regulatorias del agua y tenga como fin la garantía del derecho al agua. 
4. Los refugiados, los solicitantes de asilo, los desplazados internos y los repatriados tengan acceso al agua potable tanto si permanecen en campamentos o en las zonas urbanas y rurales.
9. Incorporar en la gestión de cuencas indicadores sobre la garantía del derecho humano al agua.
5. Los presos y detenidos tengan agua suficiente y salubre para atender a sus necesidades individuales cotidianas.
10. Integración efectiva y progresiva del saneamiento como derecho humano.

Fuente: elaboración propia a partir del contenido de la Observación General n. ${ }^{0} 15$ (Comité de Derechos Económicos, Sociales y Culturales de las Naciones Unidas, 2002) y Federación Iberoamericana de Ombudsman (2015).

De acuerdo con estas reglas, por lo menos en el contexto regional, todo proceso de gobernanza del agua debe respetar unas condiciones jurídicas mínimas. Concretamente en el caso colombiano, el Acuerdo de Escazú (Naciones Unidas, 2018), conjuntamente con los estándares del derecho al agua complementa y fortalece la regulación de la deliberación de los Consejos de Cuenca, siendo estos considerados como los espacios de la gobernanza del agua en el país.

\section{MARCO JURIDICO COLOMBIANO DE LA GOBERNANZA DEL AGUA: LOS CONSEJOS DE CUENCA}

La gobernanza del agua en Colombia se desarrolla jurídicamente, como se mencionó en la introducción, por medio del Decreto 1640 de $2012^{4}$ el cual reglamenta la PHN de 2010 en el marco del nuevo esquema de planificación hídrica que confirma a las cuencas hidrográficas como la unidad básica de gestión (Ministerio de Ambiente y Desarrollo Sostenible, 2012). Específicamente, el Decreto 1640 de 2012 crea los Consejos de cuenca (artículos 48 al 53) para cumplir con lo dispuesto en las estrategias 6.1. "Participación" y 6.3. "Manejo de Conflictos" del objetivo 6 "gobernabilidad". A su vez, los Consejos de cuenca, como instancia de participación en la ordenación y manejo de cuencas, son reglamentados por la Resolución 509 de 2013 (Ministerio de Ambiente y Desarrollo Sostenible, 2013). Por su parte, las directrices para la participación en la formulación, seguimiento y evaluación de los Pomca ${ }^{5}$ es descrita en la guía metodológica ${ }^{6}$ del 2013.

4 Compilado por el Decreto Único Ambiental 1076 de 2015.

5 Son normas de superior jerarquía que se constituyen en un determinante ambiental para la elaboración y adopción de los planes de ordenamiento territorial de acuerdo con el artículo 10 de la Ley 388 de 1997.

6 El parágrafo 1 del Artículo 26 establece que las autoridades ambientales competentes para desarrollar el Plan de ordenación y manejo de cuenca deberán seguir los criterios técnicos, procedimientos y metodologías establecidos en la guía. Esto le otorga carácter vinculante a la guía. 
Las normas citadas y la guía conforman el marco reglamentario base tanto para la creación de los Consejos de cuenca como para su desarrollo práctico. Con fundamento en un enfoque de derechos es necesario citar que ninguno de estos instrumentos menciona o relaciona sus disposiciones como garantías del derecho humano al agua. Una revisión breve permite vislumbrar los vacíos que esta normativa contiene, sobre todo siguiendo el enfoque propuesto por Madrigal (2018) y, recientemente, por el tratado de Escazú (Naciones Unidas, 2018), como se intenta a continuación.

El Decreto 1640 de 2012, en su artículo 48, define los Consejos de cuenca como: "la instancia consultiva y representativa de todos los actores que viven y desarrollan actividades dentro de la cuenca hidrográfica". En estos términos, los consejos serán conformados, según el artículo 49, por: "los representantes de cada una de las personas jurídicas públicas y/o privadas asentadas y que desarrollen actividades en la cuenca, así como de las comunidades campesinas, indígenas y negras, y asociaciones de usuarios y gremios según sea el caso".

Lastimosamente, el Decreto 1640 de 2012 deja de lado la obligación de las autoridades públicas de contribuir con la promoción y fortalecimiento de la participación como lo señalan los estándares del Acuerdo de Escazú. Esto se evidencia claramente en el parágrafo del artículo 48, que señala: "la autoridad ambiental competente podrá apoyar los aspectos logísticos y financieros del Consejo de cuenca". Sobre la existencia o no de financiamiento público para los Consejos cuenca, el Ministerio de Ambiente y Desarrollo Sostenible fue consultado por medio de petición, y en su respuesta afirmó que las autoridades ambientales han venido apoyando la elección y conformación de los mismos, pero que:

Es responsabilidad de los Consejos de cuenca establecer un reglamento interno en el que deberán incluirse aspectos relacionados al funcionamiento del consejo, en tal sentido dicho reglamento debe incluir un plan de acción o de trabajo que incorpore una estrategia de sostenibilidad en el que se contemplen líneas estratégicas de tipo financiero, teniendo en cuenta que todos los actores son corresponsables del funcionamiento y fortalecimiento de dicha instancia de participación (Ministerio de Ambiente y Desarrollo Sostenible, 2018).

Con lo anterior, es claro que para las autoridades ambientales los Consejos de cuenca deben autofinanciarse, dejando a un lado la obligación del Estado de brindar apoyo logístico y financiero, especialmente en los escenarios de participación recientemente creados y con el fin primordial de integrar a la población en condición de vulnerabilidad. Por ello, se deben definir puntualmente las responsabilidades públicas desde un enfoque diferencial, en particular en torno a los grupos en condición de vulnerabilidad. De lo contrario, la participación se verá limitada y no aportará a prevenir los conflictos sino que, por el contrario, los aumentará, porque no se sabrá en qué espacios, con qué equipos y materiales va a sesionar el Consejo de cuenca y 
por qué posiblemente existirán actores relevantes que no cuenten con los recursos financieros para acudir a las reuniones, generando también una subrepresentación, ya que los aportes del Consejo de cuenca no representan efectivamente la visión, deseo y conocimiento de los actores clave de la cuenca.

Sobre los miembros de los Consejos de cuenca y sus funciones, se establece que estarán por un período de cuatro años contados a partir de su instalación y entre sus funciones se destacan, de acuerdo con el artículo 50, las siguientes:

Participar en las fases del Plan de Ordenación de la cuenca de conformidad con los lineamientos que defina el Ministerio de Ambiente y Desarrollo Sostenible; servir de canal para la presentación de recomendaciones y observaciones en las diferentes fases del proceso de ordenación y manejo de la cuenca hidrográfica declarada en ordenación, por parte de las personas naturales y jurídicas asentadas en la misma; divulgar permanentemente con sus respectivas comunidades o sectores a quienes representan, los avances en las fases del proceso de ordenación y manejo de la cuenca; y contribuir con alternativas de solución en los procesos de manejo de conflictos en relación con la formulación o ajuste del plan de ordenación y manejo de la cuenca hidrográfica y de la administración de los recursos naturales renovables de dicha cuenca (Ministerio de Ambiente y Desarrollo Sostenible, 2012).

De la conformación de los consejos se desprende que las personas naturales no pueden ser miembros del consejo, pero al igual que las personas jurídicas públicas o privadas que se encuentren asentadas en la cuenca y que no hagan parte del consejo, pueden participar en las diferentes fases de los planes de ordenamiento y manejo de cuencas, enviando sus recomendaciones y observaciones por medio de los representantes en el Consejo de cuenca correspondiente, de acuerdo con lo establecido en el artículo 53 del Decreto 1640 de 2012. Este aspecto, si bien no vulnera directamente el estándar del Acuerdo de Escazú, relacionado con la participación abierta e inclusiva, sí limita la participación, pues no permite que todos los actores sean parte del consejo, lo cual se podría comprender como una medida de gestión y optimización del espacio mismo de participación orientada a la organización y cumplimiento de las funciones, pudiéndose armonizar con la creación de redes externas articuladas con el consejo que permitan una mayor representatividad de los intereses reales de los actores sociales que habitan la cuenca.

También debe resaltarse que el alcance de la participación del Consejo de cuenca en las fases del Pomca no se define; no se establecen los procedimientos para que los aportes del consejo tengan incidencia en las decisiones de las autoridades, como lo son: audiencias o asambleas para la presentación de los aportes por cada fase de ordenación y manejo de la respectiva cuenca que integren a los miembros del Consejo de cuenca con la autoridad ambiental o los miembros de la comisión conjunta. La creación de canales o medios de comunicación continuos y abiertos entre los espacios 
de participación en asuntos hídricos y la autoridad ambiental o comisión conjunta que permitan atender la participación ciudadana en debida forma. Y, por último, la consagración de la obligatoriedad de respuesta motivada por parte de la autoridad ambiental competente sobre la integración o no, total o parcial de los aportes del Consejo de cuenca, de conformidad con lo establecido en el artículo 23 constitucional y de los principios consagrados en la Ley 1757 de 2015 sobre participación democrática.

En torno a la Resolución 509 de 2013, es importante mencionar inicialmente que esta se deriva del mandato establecido por el parágrafo del artículo 49 del Decreto 1640 de 2012 que establece que: "El Ministerio de Ambiente y Desarrollo Sostenible en un plazo no mayor a tres meses, contados a partir de la publicación del presente decreto, establecerá lineamientos para su conformación". Por ello, la mencionada resolución, integrada por diez artículos, tiene como objeto: "Establecer los lineamientos para la conformación de los Consejos de Cuenca y su participación en las fases del Plan de Ordenación de la Cuenca" (Ministerio de Ambiente y Desarrollo Sostenible, 2013).

La Resolución 509 (Ministerio de Ambiente y Desarrollo Sostenible, 2013) incluye tres temas principales: el primero aborda a la descripción de los miembros del consejo y la manera como se eligen; el segundo habla sobre el funcionamiento y, el tercero, sobre la participación del consejo en las fases del Pomca ${ }^{7}$. Sobre el primer tema, relacionado con la conformación, se destaca que no se incluyen a las autoridades ambientales dentro del Consejo de cuenca, al parecer para buscar una mayor autonomía, lo que evita la interacción y el ejercicio deliberativo, que a todas luces define la gobernanza ambiental.

Sobre la integración de otros actores en el consejo, existen movimientos y colectivos ambientales que llevan a cabo actividades muy importantes y con incidencia en la cuenca, que, al no tener personería jurídica, no pueden ser miembros del consejo, al igual que las agrupaciones juveniles ambientalistas. La exigencia de personería jurídica se encuentra establecida desde los artículos tercero al sexto, que reglan el procedimiento para elegir a los miembros del consejo. En este punto, vale la pena destacar que, en la negociación del tratado de Escazú, fue claro que no puede limitarse la participación de colectivos u organizaciones ambientales a aquellas que tenga una forma jurídica reconocida, debido a las limitaciones económicas que ello supone para muchas comunidades en los países de la región. Por esta razón, el artículo dos, sobre definiciones, dispone que se entenderá por público: "una o varias personas físicas o jurídicas y las asociaciones, organizaciones o grupos constituidos por esas personas, que son nacionales o que están sujetos a la jurisdicción nacional del Estado parte" (Naciones Unidas, 2018, p. 4).

\footnotetext{
7 Las fases de acuerdo con el artículo 26 del Decreto 1640 de 2012 son: aprestamiento, diagnóstico,
} prospectiva y zonificación ambiental, formulación, ejecución, seguimiento y evaluación. 
En cuanto al segundo tema -relacionado con el funcionamiento-, la resolución únicamente desarrolla un artículo en el cual se aborda la elección del presidente y la secretaría, y la elaboración del reglamento interno, el cual precisamente deberá describir el funcionamiento del consejo.

Por último, en cuanto al tercer asunto sobre la participación del consejo en las fases del Pomca, se resalta que en la Resolución 509, pese a ser esencialmente reglamentaria, no se definen mecanismos ni procedimientos que contribuyan al acceso de la información de manera oportuna, clara y completa, lo que vulnera otro de los estándares básicos del Acuerdo de Escazú, relacionado con el acceso a la información en los procesos de participación. Esto es especialmente importante si se considera la diversidad socio-cultural de los miembros del Consejo de cuenca y la calidad técnica de la información generada en torno a la ordenación y manejo de las cuencas. En la resolución tampoco se describen las medidas de apoyo a personas o grupos en condición de vulnerabilidad de manera tal que sean involucradas de manera activa, oportuna y efectiva en el Consejo de cuenca, un estándar también reconocido por el citado instrumento regional.

Aquí es conveniente citar que el mencionado acuerdo considera como "personas o grupos en situación de vulnerabilidad" a:

Aquellas personas o grupos que encuentran especiales dificultades para ejercer con plenitud los derechos de acceso reconocidos en el presente Acuerdo, por las circunstancias o condiciones que se entiendan en el contexto nacional de cada Parte y de conformidad con sus obligaciones internacionales (Naciones Unidas, 2018, p. 4).

Vinculado al procedimiento de los Consejos de cuenca, es relevante conocer que la guía técnica está integrada por una serie de criterios, procedimientos y metodologías que son de carácter obligatorio para las autoridades ambientales competentes al momento de desarrollar las fases de ordenación y manejo de cuencas en su jurisdicción, de conformidad con lo establecido en el artículo 26 del Decreto 1640 de 2012. Además de lo anterior, se destaca que genera las bases para delimitar el alcance de la gobernanza del agua, mencionando que para la formulación de los planes de ordenación y manejo de cuencas se debe reconocer que los intereses de los diversos actores son relevantes $\mathrm{y}$, por lo tanto, se requiere de:

La consideración justa y equitativa de las necesidades y responsabilidades existentes en la cuenca, lo que hace imprescindible el establecimiento de procedimientos transparentes, para la consideración motivada de las recomendaciones de los actores clave, lo que constituirá la base de confianza de las interacciones de las personas que intervienen (Ministerio de Ambiente y Desarrollo Sostenible, 2014, p. 14). 
Y que establece -como el principal instrumento para el desarrollo adecuado de la gobernanza del agua- la estrategia de participación que deberá crearse para cada una de las fases del plan de ordenación y manejo de cuencas, orientando especialmente a los Consejos de cuenca y a las propias autoridades ambientales.

La guía técnica confirma el enfoque, principalmente instrumental, de la gobernanza del agua consagrado en el Decreto 1640 de 2012 y en la Resolución 509 de 2013, esto se sustenta en que: i) se direcciona y reglamenta la gobernanza del agua por medio de la estrategia de participación, la cual define la manera como deben participar los actores sociales y los resultados del proceso de participación, ii) los Consejos de cuenca cuentan con diversas funciones (obligaciones) pero sin financiamiento directo, iii) la no determinación del alcance de la participación, lo cual vulnera este derecho, principalmente para el caso de los grupos marginados y movimientos de base, quienes deberían ser integrados de manera efectiva, continua y diferencial, iv) la carencia de mecanismos particulares de acceso a la información, transparencia y rendición de cuentas para el proceso de ordenación y manejo de cuenca y v) el excesivo formalismo en torno a la conformación e integración de nuevos miembros restringe la posibilidad de fortalecer el espacio de participación con nuevos aportes y la distribución de responsabilidades.

\section{CONCLUSIONES}

El reconocimiento jurídico del proceso de gobernanza en la gestión de cuencas y de una instancia operativa del proceso, como los Consejos de cuenca, abre un nuevo escenario para la participación de la sociedad civil en los asuntos hídricos. Sin embargo, el enfoque propuesto por Madrigal (2018) y los estándares sobre participación en asuntos ambientales definidos en el Acuerdo de Escazú posibilitan una interpretación del marco reglamentario básico que valora el derecho humano al agua en la ordenación y manejo de cuencas desde la integración de estándares sobre participación que, bajo una relación dialéctica (medio-fin) con el mencionado derecho, dotan a la gobernanza del agua de un mayor contenido normativo, lo cual es fundamental en contextos de alta conflictividad como el colombiano.

Así, los Consejos de cuenca deben ser valorados como el espacio propicio para la prevención y manejo integral de los conflictos por el agua, generando para ello actividades relacionadas con la cultura del agua y procedimientos para el manejo alternativo de los conflictos por el agua, adecuados a su territorio.

Con base en un enfoque basado en la garantía de derechos, las autoridades ambientales deben de manera coordinada con el Ministerio de Ambiente y Desarrollo Sostenible mejorar la reglamentación de los Consejos de cuenca, especialmente 
en aspectos como el acceso a la información, la integración de nuevos consejeros una vez conformado el consejo, el diseño e implementación de acciones afirmativas a favor de los grupos marginados y sujetos a protección especial y la incorporación del componente de deliberación como pilar de la gobernanza hídrica. En este orden de ideas, los modelos de gobernanza en materia hídrica que son de iniciativa estatal deben ser flexibles y abiertos, y ser una entre distintas opciones; la normatividad y políticas públicas deben permitir modelos de gobernanza hídrica propuestos por los actores sociales, especialmente si son sujetos de protección especial.

No obstante, si no se garantiza el financiamiento permanente de los Consejos de cuenca, por lo menos para su gasto operacional y generar así la posibilidad de concentrar los esfuerzos en las funciones específicas de los Consejos, nada de lo dicho anteriormente tiene verdaderas posibilidades de aplicación. Opciones como las corporaciones públicas sin ánimo de lucro permitirían a los consejos canalizar y administrar autónomamente recursos financieros de diversas instancias nacionales e internacionales.

En todo caso, la reglamentación de la gobernanza del agua en Colombia no debe ceñirse a los Consejos de cuenca, debe ampliarse y promover la integración de procesos desde abajo, comunitarios y tradicionales, favoreciendo de esta manera la concertación y respetando las formas históricas de autogestión del agua. La integración de los estándares del Acuerdo de Escazú y del reconocimiento de la importancia de las personas y grupos en condición de vulnerabilidad, lejos de dotar a la gobernanza del agua de criterios asistencialistas, busca cumplir efectivamente con la participación en su faceta derecho y deber, ajustándose a la realidad socioeconómica y cultural del país. Por ello, todo lo relacionado con la transparencia y acceso a la información también se aplica a los Consejos de cuenca, y también es su deber trabajar en el mejoramiento integral de la gobernanza del agua, ojalá desde el marco propuesto, esto es considerando los derechos humanos de manera amplia y diversa.

\section{REFERENCIAS}

Ballester, A. y La Calle, A. (2015). Gobernanza del agua. Participación pública en la planificación hidrológica (Cuadernos Prácticos n. 5). Recuperado de https://fnca.eu/images/documentos/ ODMA/4a\%20FASE/guia_oppa_participacion.pdf

Castro-Buitrago, E. (2017). Derecho ambiental y gobernanza: la concertación como actuación informal a la administración. Medellín: Universidad de Medellín.

Castro-Buitrago, E. y Calderón, F. (2018). Un derecho ambiental democrático para Latinoamérica y el Caribe: los retos de la negociación del Acuerdo Regional sobre el Principio 10 de Río 92. Anuario Colombiano de Derecho Internacional, 11, 159-186. 
Cerrillo, A. (2005). La gobernanza hoy: introducción. En A. Cerrillo (Coord.), La gobernanza hoy: 10 textos de referencia (pp. 11-36). Madrid: Instituto Nacional de Administración Pública.

Cuadrado-Quesada, G. (2017). Gobernanza de aguas subterráneas, conflictos socioambientales y alternativas: experiencias de Costa Rica. Anuario de Estudios Centroamericanos, 43, 393-418.

Cepal. (2018). Antecedentes del acuerdo regional. Recuperado de https://www.cepal.org/es/organossubsidiarios/acuerdo-regional-acceso-la-informacion-la-participacion-publica-acceso-la/ antecedentes-acuerdo-regional

Comisión Europea. (2001). El libro blanco sobre la gobernanza europea. Recuperado de http://eur-lex. europa.eu/LexUriServ/LexUriServ.do?uri=OJ:C:2001:287:0001:0029:ES:PDF

Comité de Derechos Económicos, Sociales y Culturales de las Naciones Unidas. (2002). Observación general $N^{\circ}$ 15: el derecho al agua (artículos 11 y 12 del Pacto Internacional de Derechos Económicos, Sociales y Culturales). $29^{\circ}$ período de sesiones. Ginebra, 11 a 29 de noviembre. Recuperado de https://www.escr-net.org/es/recursos/observacion-general-no-15-derecho-alagua-articulos-11-y-12-del-pacto-internacional

Congreso de la República de Colombia. (1993). Ley 99 de diciembre 22 por la cual se crea el Ministerio del Medio Ambiente, se reordena el sector público encargado de la gestión y conservación del medio ambiente y los recursos naturales renovables, se organiza el Sistema Nacional Ambiental, SINA y se dictan otras disposiciones. Diario Oficial 41.146 de diciembre 22 de 1993.

Consejo Mundial del Agua. (2012). Hacia una buena gobernanza para la gestión integrada de los recursos hídricos. Recuperado de http://www.oas.org/en/sedi/dsd/IWRM/Past\%20Events/D7/6\%20 WWF-GOBERNANZA\%20Final.pdf

Corte Constitucional. Colombia. (2010). Sentencia C 293 de abril 21. M. P. Pinilla, N.

De Sousa Santos, B. y Rodríguez, C. (2007). El derecho, la política y lo subalterno en la globalización contrahegemónica. En B. De Sousa Santos y C. Rodríguez (Eds.), El derecho y la globalización desde abajo: hacia una legalidad cosmopolita (pp. 7-28). Ciudad de México: Anthropos.

Domínguez, J. (2006). La gobernanza del agua en México y el reto de la adaptación en zonas urbanas: el caso de la ciudad de México. Anuario de Espacios Urbanos, Historia, Cultura y Diseño, (13), 273-296.

Dourojeanni, A. y Jouravlev, A. (2001). Crisis de gobernabilidad en la gestión del agua (desafíos que enfrenta la implementación de las recomendaciones contenidas en el capítulo 18 del Programa 21). Recuperado de https://archivo.cepal.org/pdfs/Waterguide/TEXT/LETTERS/list_cd/LCL1660S.pdf

Federación Iberoamericana de Ombudsman. (2015). Derecho al agua: XII informe sobre derechos humanos. Madrid: Trama.

Instituto de Hidrología, Meteorología y Estudios Ambientales. Ideam. (2015). Estudio nacional del agua 2014. Recuperado de http://documentacion.ideam.gov.co/openbiblio/bvirtual/023080/ ENA_2014.pdf 
Instituto Geográfico Agustín Codazzi. IGAC. (2014). Colombia estrenará estudio de sus cuencas hidrográficas. Recuperado de http://sitio.fondoadaptacion.gov.co/index.php/prensa/comunicadosde-prensa/comunicados-prensa-2014/372-colombia-estrenara-estudio-de-sus-cuencashidrograficas

Jacobi, P., Silva-Sánchez, S. y Fracalanza, A. (2015). Gobernanza del agua en la ciudad de Sao Paulo: actores sociales, degradación socioambiental y acciones públicas innovadoras. América Latina Hoy, 69, 35-51.

Querejazu-Escobari, A. (2016). Violencias encubiertas de la gobernanza global. Estudios Políticos, (49), 148-166.

Madrigal, M. (2018). Gobernanza hídrica en la cuenca del río Aburrá: un análisis del derecho humano al agua y el Consejo de cuenca (2012-2017) (Tesis de maestría). Universidad de Antioquia, Medellín.

Mello, F. y Villar, P. (2015). Gobernanza de las aguas transfronterizas: fragilidades institucionales en América del Sur. América Latina Hoy, 69, 53-74.

Ministerio del Medio Ambiente. (2002). Decreto 1729 agosto 6 por el cual se reglamenta la Parte XIII $<$ sic >, Título 2, Capítulo III del Decreto-ley 2811 de 1974 sobre cuencas hidrográficas, parcialmente el numeral 12 del artículo 5o. de la Ley 99 de 1993 y se dictan otras disposiciones. Diario Oficial 44.893 de 07 de agosto de 2002.

Ministerio de Ambiente, Vivienda y Desarrollo Territorial. (2010). Política nacional para la gestión integral del recurso hídrico. Recuperado de http://www.catedras-bogota.unal.edu.co/catedras/ gaitan/2016-I/gaitan_2016_I/docs/lecturas/s07/politica.pdf

Ministerio de Ambiente y Desarrollo Sostenible. (2012). Decreto 1640 de agosto 2 por medio del cual se reglamentan los instrumentos para la planificación, ordenación y manejo de las cuencas hidrográficas y acuíferos, y se dictan otras disposiciones. Diario Oficial 48.510 de agosto 2 de 2012.

Ministerio de Ambiente y Desarrollo Sostenible. (2013). Resolución 0509 de mayo 21 por la cual se definen los lineamientos para la conformación de los Consejos de cuenca y su participación en las fases del Plan de Ordenación de la Cuenca y se dictan otras disposiciones. Diario Oficial 48.803 de mayo 27 de 2013.

Ministerio de Ambiente y Desarrollo Sostenible. (2014). Guía técnica para la formulación de los planes de ordenación y manejo de cuencas hidrográficas. Recuperado de http://www.minambiente.gov. co/images/GestionIntegraldelRecursoHidrico/pdf/cuencas-hidrograficas/GUIA_DE_POMCAS.pdf

Ministerio de Ambiente y Desarrollo Sostenible. (2015). Decreto 1076 de mayo 26 por medio del cual se expide el decreto único reglamentario del sector ambiente y desarrollo sostenible. Diario Oficial 49.523 de mayo 26 de 2015.

Ministerio de Ambiente y Desarrollo Sostenible. (2017). Gobernanza del agua. Recuperado de http:// www.minambiente.gov.co/index.php/gestion-integral-del-recurso-hidrico/gobernanza-del-agua 
Ministerio de Ambiente y Desarrollo Sostenible (2018). Respuesta a derecho de petición: solicitud de información ambiental. Referencia: DGI - 8230 -E2-2018-038596, 26 de diciembre de 2018. Derecho de petición elaborado y presentado por Mauricio Madrigal.

Mussetta, P. (2009). Participación y gobernanza: el modelo de gobierno del agua en México. Espacios Públicos, 12(25), 66-84.

Naciones Unidas. (2018). Acuerdo regional sobre el acceso a la información, la participación pública y el acceso a la justicia en asuntos ambientales en América Latina y el Caribe. Recuperado de https://www.cepal.org/es/acuerdodeescazu

Pacheco-Vega, R. (2015). Gobernanza del agua residual en Aguascalientes: captura regulatoria y arreglos institucionales complejos. Región y sociedad, 27(64), 313-350.

Parés, M., Ballester, A., Espluga, J. y Brugué, Q. (2014). Gobernanza deliberativa en la gestión de cuencas hidrográficas: analizando las consecuencias de la Directiva Marco del Agua en Cataluña. Investigaciones Geográficas, (61), 113-127.

Porras, F. (2016). Gobernanza: propuestas, límites y perspectivas. Ciudad de México: Editorial Mora.

Presidencia de la República. (1981). Decreto 2857 de octubre 13 por el cual se reglamenta la Parte XIII, Capítulo III del Decreto Ley 2811 de 1974 sobre cuencas hidrográficas y se dictan otras disposiciones. Diario Oficial 35.881 de noviembre 10 de 1981.

Presidencia de la República. (2012). Decreto 1640 de agosto 2 por medio del cual se reglamentan los instrumentos para la planificación, ordenación y manejo de las cuencas hidrográficas y acuíferos, y se dictan otras disposiciones. Diario Oficial 48510 de agosto 2 de 2012.

Redacción Semana. (2010). Cifra de damnificados por el invierno se acerca a los dos millones. Recuperado de http://www.semana.com/nacion/articulo/cifra-damnificados-invierno-acercados-millones/125898-3

Santa, J. (2014). Ciudad, poder y gobernanza: el acceso al agua potable en Medellín, 2002-2011 (Tesis de maestría). Universidad Nacional de Colombia, Medellín.

Torres, G. y Ramos, A. (2012). Nueva gestión de lo local. Desarrollo rural y construcción de sistemas agroalimentarios. En B. Lerner, R. Uvalle y R. Moreno (Coords.), Gobernabilidad y gobernanza en los albores del siglo XXI y reflexiones sobre el México contemporáneo (pp. 99-136). Toluca: Universidad Nacional Autónoma de México e Instituto de Administración Pública del Estado de México. 